\title{
Numerical Simulation on the Surface Layer Hardness of 40Cr Steel in Grind-Hardening
}

\author{
Tian-Cheng HUANG ${ }^{1, a}$, Jin-Yi ZHANG ${ }^{2, b}$, Gui-Cheng WANG ${ }^{1,2, c^{\star}}$ \\ ${ }^{1}$ Nantong Institute of Technology, Nantong, Jiangsu, 226002 P. R. China \\ ${ }^{2}$ School of Mechanical Engineering, Jiangsu University, Zhenjiang, Jiangsu, 212013 P.R. China \\ ahuangtc2000@163.com, bjyzhang@ujs.edu.cn, cwgch@ujs.edu.cn \\ *Corresponding author
}

Keywords: $40 \mathrm{Cr}$ steel, Grind-hardening, Temperature field, Microstructure field, $40 \mathrm{Cr}$ Steel, Surface layer, Simulation, Prediction

\begin{abstract}
Grind-hardening is not only a complex process coupling mechanical, dynamical and thermal effects, but a process containing distinct changes of microstructure and performances in the surface layer of the grinded workpiece. Based on the software Deform-2D, this paper simulated the distribution and variation of the fields of temperature, microstructure and micro-hardness in the hardened layer of 40Cr. The micro-hardness of the hardened layer was obtained, which coincided well with the experimental results. The work here has provided a new way for making and implementing the grind-hardening process, as well as for scientifically and generally evaluating the effects of grind-hardening.
\end{abstract}

\section{Introduction}

Grind-hardening is a new process that utilizes the composite thermal and mechanical effects of grinding to directly quench the surface layer of non-hardened steels. It not only integrates grinding and surface quenching, but reduces investments of thermal treatment equipment and production people, as well as the environmental pollution from emissions, so it has remarkable economic and social benefits [1].

Brinksmeier and Zarudi etc. had indicated that rough grinding could produce martensitic hardened layer characterized by compressive residual stresses and no surface cracks. The specific microstructure and properties of the hardened layer formed from the combined functions of the heavy gradient fields of temperature and stress and strain $[2,3,4,5]$.

Grind-hardening couples the fields of temperature, stress, strain and microstructure, and many parameters such as material properties show strong non-linear characters, which make the problem quite complicated. Experimental investigation is a reliable method, but the costs are high. Finite element method (FEM) is an effective tool to solve non-linear and multiple physical field coupling problems, so it has been widely used in engineering analyses. To analyze the forming process of the hardened layer, evaluate hardened effects, and control hardened qualities, based on grinding experiments, this paper simulated the fields of temperature, microstructure and micro-hardness in grind-hardening.

\section{Grind-hardening Experiments}

The testing material is $40 \mathrm{Cr}$, initially tempered, with dimension of $79 \mathrm{~mm} \times 4 \mathrm{~mm} \times 15 \mathrm{~mm}$ (length $\times$ width $\times$ height). The grinding experiments were performed on a precision surface grinder (MMD7125) using conditions listed in Tab.1.Grinding forces were measured by a dynamometer (Kistler 9265B). The characters of macro- and microstructure of the hardened layer were observed on a SEM (JXA-840A), and the micro-hardness was measured by a micro-hardness tester (HVS-1000). 
Tab.1. Experimental conditions for grinding 40Cr

\begin{tabular}{|l|l|}
\hline Original microstucture & Tempered sorbite \\
\hline Grinding wheel & P300×40×127 A60L6V \\
\hline Grinding speed $\mathrm{V}_{\mathrm{s}}$ & $25(\mathrm{~m} / \mathrm{s})$ \\
\hline Grinding depth $\mathrm{a}_{\mathrm{p}}$ & $0.2(\mathrm{~mm})$ \\
\hline Feed speed $\mathrm{V}_{\mathrm{w}}$ & $0.4(\mathrm{~m} / \mathrm{min})$ \\
\hline Grinding manner & Single pass surface plunge grinding \\
\hline Cooling condition & Dry grinding \\
\hline
\end{tabular}

\section{Setting Up the Finite Element Model}

Before solving, a finite element model of the problem should be established in the pre-processor of Deform-2D.

\section{Setting Up the Geometrical Model and Generating Mesh}

Because the width of the workpiece is small and no coolant is used, heat exchange between side faces of the workpiece and atmosphere is low, making the temperature gradient in the direction of width small. Therefore, a 2-D model is used here to substitute the 3-D model.

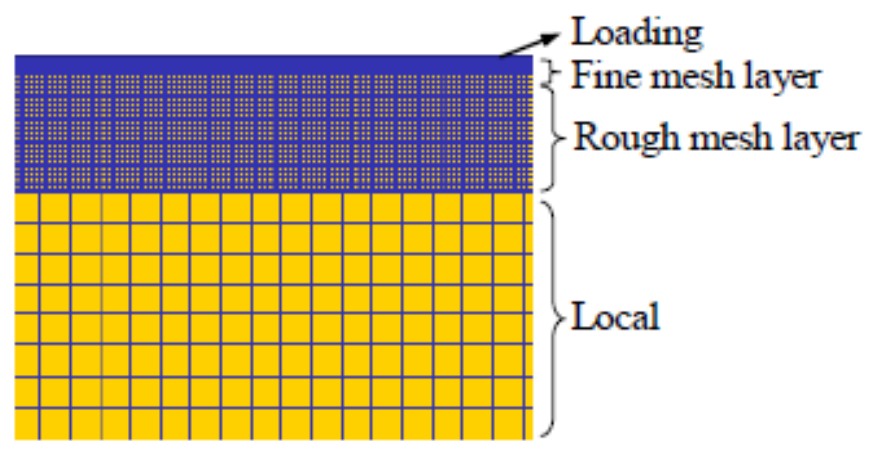

Fig. 1.Geometrical model with mesh

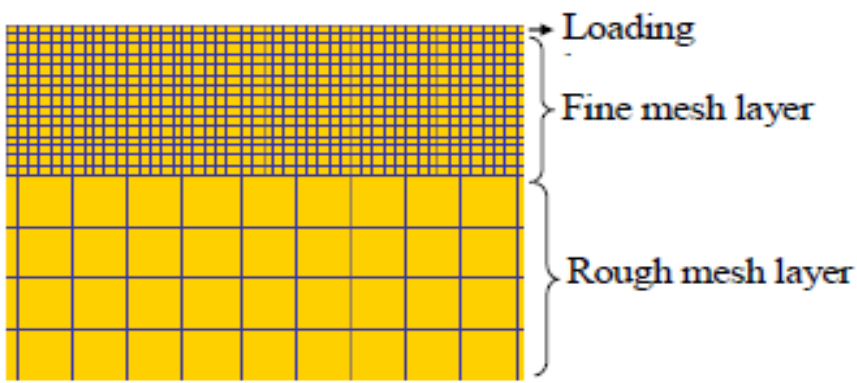

Fig. 2. Selective enlargement of the model

Weighing both computational complexity and precision, as well as facilitating compiling subroutines of thermal boundary conditions, the workpiece is divided into three independent layers, namely the loading layer, the fine mesh layer and the rough mesh layer. The side lengths of square elements selected for the three layers and the worktable are $0.1,0.1,0.5$ and 10/3mm respectively. Fig. 1 shows the geometrical model with mesh and the selective enlargement is shown in Fig.2.

\section{Determining Material Properties}

\section{Determining Material Properties for Workpiece and Worktable}

The workpiece is treated as a multiphase body containing phases that might appear in grinding [9]: $\mathrm{S}$ (tempered sorbite), A (austenite), $\mathrm{F}+\mathrm{P}$ (mixture of ferrite and pearlite), $\mathrm{F}+\mathrm{P}+\mathrm{B}$ (mixture of ferrite, 
pearlite and bainite), B (bainite) and $\mathrm{M}$ (martensite). The worktable is treated as cast iron. Utilizing literature [6-11] and the material property library of Deform-2D, parameters such as thermal conductivity, heat capacity, emissivity, density and micro-hardness could be determined.

\section{Determining Phase Transformation Kinetics Models}

$\mathrm{S} \rightarrow \mathrm{A}$ belongs to the diffusional phase transformation that could be described by a simplified model [12]:

$$
\xi_{S \rightarrow A}=1-\exp \left[A_{1}\left(\frac{T-T_{s}}{T_{e}-T_{s}}\right)^{A_{2}}\right]
$$

Where $A_{1}$ and $A_{2}$ are constants, here assigned -4 and 2. $T_{s}$ and $T_{e}$ are the beginning and finishing temperatures for the austenitic transformation, which increase with acceleration of heating velocity[13]. Supposing the average heating velocity is $800^{\circ} \mathrm{C} / \mathrm{s}$ (the simulation results supporting this assumption), they could be assigned $775^{\circ} \mathrm{C}$ and $865^{\circ} \mathrm{C}$ respectively.

$\mathrm{A} \rightarrow \mathrm{M}$ belongs to the diffusionless phase transformation that the transformation amount only depends on temperature and is independent of time [14]:

$$
\xi_{A \rightarrow M}=1-\exp \left[-\alpha\left(M_{s}-T\right)\right]
$$

Where $\alpha$ is a constant that reflects the transformation velocity of martensite and changes with material composition, here assigned 0.011. $M_{s}$ is the beginning temperature of transformation, here assigned $325^{\circ} \mathrm{C}[9] . \mathrm{A} \rightarrow \mathrm{F}+\mathrm{P}, \mathrm{A} \rightarrow \mathrm{F}+\mathrm{P}+\mathrm{B}$ and $\mathrm{A} \rightarrow \mathrm{B}$ belong to diffusional or semi-diffusional phase transformations. When continuously cooling, based on the Avrami equation, using the isothermal transformation curve and Scheil's superposition laws, the transformation amount for the three phases could be calculated [14].

The Avrami equation describes the relationship between transformation amount and time:

$$
\xi=1-\exp \left(-b t^{n}\right)
$$

Where $t$ is isothermal time. Using the TTT curve function, two time $t_{1}$ and $t_{2}$ corresponding with two austenitic phase transformation amount $\xi_{1}=1 \%$ and $\xi_{2}=50 \%$ at a certain temperature $T$ can be calculated, and then $b$ and $n$ can be obtained:

$$
n=\frac{\ln \left\{\left[\ln \left(1-\xi_{1}\right)\right] /\left[\ln \left(1-\xi_{2}\right)\right]\right\}}{\ln \left(t_{1} / t_{2}\right)} \quad b=\frac{-\ln \left(1-\xi_{1}\right)}{t_{1}^{n}}
$$

The TTT curve function can be attained by an approach as follows: firstly divide the curve [9] into three regions according to temperature, namely $712 \sim 538^{\circ} \mathrm{C}$ corresponding with the $\mathrm{A} \rightarrow \mathrm{F}+\mathrm{P}$ region, $536 \sim 447^{\circ} \mathrm{C}$ with the $\mathrm{A} \rightarrow \mathrm{F}+\mathrm{P}+\mathrm{B}$ region and $445 \sim 326^{\circ} \mathrm{C}$ with the $\mathrm{A} \rightarrow \mathrm{B}$ region; then in each region, impose the least square method on polynomial fitting the two curves of $\xi_{1}$ and $\xi_{2}$.

When continuously cooling, transformation amount is calculated basing on Scheil's superposition laws, which employ discrete time steps to change a continuous cooling process into a stepped one, and then use the Avrami equation at each discrete time step. Calculation is divided into two stages. Firstly, judge whether the incubation period ends or not: the isothermal time at a certain temperature divided by the incubation time at that temperature is the incubation ratio, and ratios at different temperatures can be added up. When summation reaches 1 , the incubation period ends and calculation steps into the second stage. Secondly, calculate transformation amount: if total transformation amount had been $V_{i}$ when temperature cooled down to $T_{i}$, virtual time $t^{*}{ }_{i+1}$ is used to convert $V_{i}$ effectively into time at temperature $T_{i+1}$. Adding virtual time to the isothermal time $d t_{i+1}$ at $T_{i+1}$, the transformation amount $V_{i+1}$ at $T_{i+1}$ can be obtained: 


$$
t_{i+1}^{*}=\left[\frac{-\ln \left(1-V_{i}\right)}{b_{i+1}}\right]^{\frac{1}{n_{i+1}}} \quad V_{i+1}=1-\exp \left[-b_{i+1}\left(t_{i+1}^{*}+d t_{i+1}\right)^{n_{i+1}}\right]
$$

Calculating phase transformation amount of $\mathrm{F}+\mathrm{P}, \mathrm{F}+\mathrm{P}+\mathrm{B}$ and $\mathrm{B}$ can't be realized unless using subroutines of Deform-2D.

\section{Determining Boundary Conditions}

\section{Determining Heat Transfer Coefficients on Interfaces between Objects}

In 3.1, the whole workpiece is divided into three layers. To make results the same as that using a complete one, other than setting the same material properties for each layer, it is necessary to set a tremendous heat transfer coefficient on interfaces between layers to make no thermal resistance and the temperature continuous. Meanwhile, on the interface between the workpiece and worktable, set the coefficient to be $3787.9(\mathrm{~W} \cdot \mathrm{m}-2 \cdot \mathrm{K}-1)[8]$.

\section{Heat Transfer by Convection and Radiation}

The free boundaries of workpiece and worktable exchange heat by convection and radiation with environment. Utilizing the correlation of the Nusselt number on both horizontal and vertical boundaries[8,10], coefficients of convective heat transfer at different temperatures can be calculated, and then functions can be obtained by data fitting:

Vertical boundaries:

$$
h_{v}=0.985+2.564 \ln t_{w}
$$

Horizontal boundaries:

$$
h_{h}=-0.542 \bar{t}_{w}^{4}+4.358 \bar{t}_{w}^{3}-12.271 \bar{t}_{w}^{2}+13.793 \bar{t}_{w}+4.5398
$$

Where $\bar{t}_{w}=t_{w} / 477.1429$

The heat flux density of convection heat transfer between boundaries and environment is given by:

$$
q_{d}=h\left(t_{w}-t_{\infty}\right)
$$

Where $t_{w}$ denotes the boundary temperature of workpiece or worktable and $t_{\infty}$ the environmental temperature $\left({ }^{\circ} \mathrm{C}\right)$. There also exists radiation exchange between boundaries and environment. Here, the boundaries are considered enclosed in an infinitely great shell with $20^{\circ} \mathrm{C}$ wall temperature, so the heat flux density of radiation exchange is given by [10]:

$$
q_{f}=\varepsilon \sigma\left(T_{w}{ }^{4}-T_{\infty}{ }^{4}\right)
$$

Where $\varepsilon$ is the emissivity of boundaries, here assigned $0.8, \sigma$ the black radiation constant, $T_{w}$ and $T_{\infty}$ the boundary and environmental temperature $(\mathrm{K})$.

\section{Applying the Moving Heat Source}

\section{Determining the Thermal Partition Ration}

The thermal partition ratio $R_{w}$ is that proportion of the grinding power $Q_{t}$ conducted into the workpiece as heat:

$$
R_{w}=\frac{\pi}{C_{1}} b \sqrt{(\kappa \rho c)_{w}} \sqrt{V_{w}} \sqrt{l_{e}} \frac{\theta_{m}}{Q_{t}}
$$


To a triangular distribution heat source, $C_{1}$ can be assigned 3.34. $b=4 \mathrm{~mm}$ is the grinding width, $(\kappa \rho c)_{w}$ the thermal property of the workpiece, which varies with temperature and microstructure, here assigned an average value $1.5683 \times 10^{8}\left(\mathrm{~J}^{2} \cdot \mathrm{m}^{-4} \cdot \mathrm{s}^{-1} \cdot \mathrm{K}^{-2}\right) . l_{e}=7.7 \mathrm{~mm}$ is the equivalent contact length between the grinding wheel and workpiece, $\theta_{m}$ the maximal temperature on the workpiece surface in the grinding zone, here assigned $1200^{\circ} \mathrm{C}$ (the simulation results supporting this assumption). $Q_{t}=\mathrm{F}_{\mathrm{t}} \cdot \mathrm{V}_{\mathrm{s}}=745 \mathrm{~W}$, where $\mathrm{F}_{\mathrm{t}}=29.8 \mathrm{~N}$ is the measuring tangential grinding force[6]. So $R_{w}=0.5437$ is obtained.

\section{Applying the Moving Heat Source}

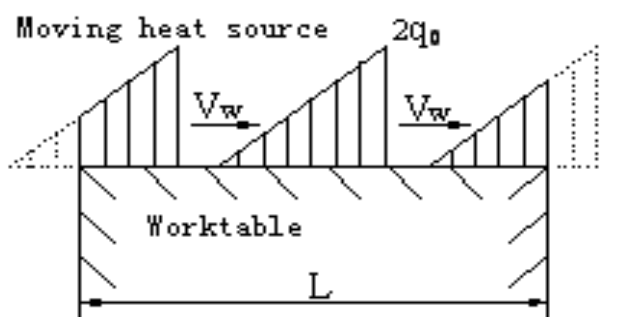

Fig.3.Scheme of the moving heat source

In the grinding zone, the distribution shape of heat source is treated as a triangle as illustrated in Figure. 3. In 3.1, the loading layer generated 790 elements and the number of which increased from 1 to 790 as counted form the left to the right. To an element $n$, when the heat source is on the elemental boundary, it only absorbs grinding heat, otherwise only exchanging heat with environment, as illustrated in equation (11) and (12). Therefore the thermal boundary conditions of the workpiece grinding surface can be described by the two equations, which can't be realized unless using subroutines of Deform-2D.

$$
\begin{gathered}
q_{z}(t)=\left\{\begin{array}{ll}
2 q_{0}\left(1-t / T_{0}\right) & 0 \leq t \leq T_{0} \\
-q_{d}-q_{f} & t>T_{0}
\end{array} \quad(n=1)\right. \\
q_{z}(t)=\left\{\begin{array}{ll}
-q_{d}-q_{f} & 0 \leq t<(n-1) t_{0} \\
2 q_{0}\left\{1-\left[t-(n-1) t_{0}\right] / T_{0}\right\} & (n-1) t_{0} \leq t \leq(n-1) t_{0}+T_{0} \\
-q_{d}-q_{f} & t>(n-1) t_{0}+T_{0}
\end{array} \quad(1<n \leq 790)\right.
\end{gathered}
$$

Where $q_{0}=R_{w} Q_{t} / b l_{e}$ is the average heat flux density, $t$ the time, $T_{0}$ and $t_{0}$ the time for the source moving a distance of an equivalent contact length and an elemental edge.

\section{Simulation Results and Discussion}

Solving the finite element model established above in the solver of Deform-2D, and then simulation results such as the distribution and alteration of the fields of temperature, microstructure and micro-hardness can be obtained in the post-processor.

\section{Distribution and Alteration Fields of Temperature and Microstructure}

From the outset of grinding to $1.76 \mathrm{~s}$, the maximal temperature in workpiece had risen to $1160^{\circ} \mathrm{C}$, and maintained $1190^{\circ} \mathrm{C}$ when the time reached $2.7 \mathrm{~s}$. Create an $\mathrm{x}$-axis in the direction of grinding originating from the left end of workpiece to facilitate analyses. After analyzing the fields of temperature and microstructure, it is clear that the region between $x=18 \mathrm{~mm}$ and $\mathrm{x}=65 \mathrm{~mm}$ in workpiece is a stable zone where the changes of variables are independent of $x$.

Selecting partial elements between $x=33.5 \mathrm{~mm}$ and $x=37.5 \mathrm{~mm}$ to analyze the fields of temperature, microstructure (distribution of volume percentage of each phase) and micro-hardness, then the whole situation in the stable zone could be known. Figure. 4 shows the temperature field at 5.76 s, and Figure. 5 
the corresponding austenitic distribution. From the figures, it can be seen that the temperature gradients in the surface layer of workpiece are quite large, with an average value of $425^{\circ} \mathrm{C} / \mathrm{mm}$; the distribution and alteration of the austenitic phase is entirely determined by the temperature field, and the front corresponds with the yellow isothermal region in Figure. 4; the workpiece has been completely austenitized above the depth of $0.8 \mathrm{~mm}$, and only partially austenitized in the narrow region of $0.9 \sim 1.2 \mathrm{~mm}$.

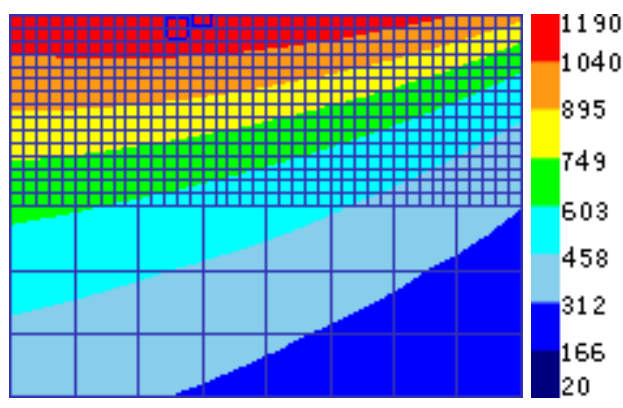

Fig.4.Temperature field at $5.76 \mathrm{~s}\left({ }^{\circ} \mathrm{C}\right)$

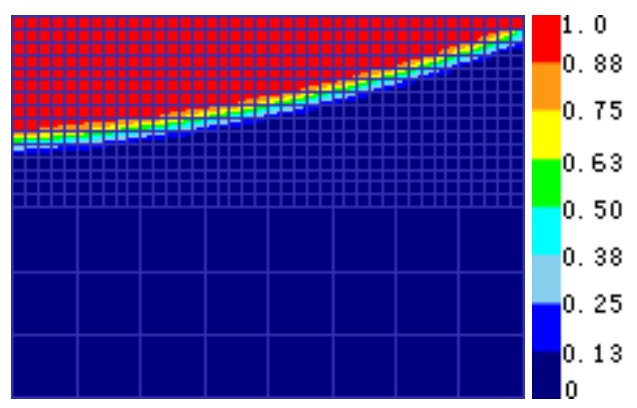

Fig.5.Austenitic distribution at 5.76s

Fig.6 shows the varying relationship between temperature and time of element 1, 8, 9 and 12 (see Fig.4). Fig.7 presents the varying relationship between warning and cooling velocities and time of element 1 (cooling velocities are negative). From the figures, it can be seen that with increase of depth, temperature response of elements lags, but they all experience six stages, namely slowly warming $\rightarrow$ quickly warming $\rightarrow$ slowly warming $\rightarrow$ slowly cooling $\rightarrow$ quickly cooling $\rightarrow$ slowly cooling; the temperature of element 1 rises from $25^{\circ} \mathrm{C}$ at $4.4 \mathrm{~s}$ to $1164^{\circ} \mathrm{C}$ at $5.8 \mathrm{~s}$, and the average and the instantaneous maximal warming velocities during this period are $814^{\circ} \mathrm{C} / \mathrm{s}$ and $5150^{\circ} \mathrm{C} / \mathrm{s}$ respectively; the cooling velocities are relatively slow, though the instantaneous maximal cooling velocity could reach as much as $-900^{\circ} \mathrm{C} / \mathrm{s}$. So the process of temperature varying in the surface layer is a quickly warming and cooling one.

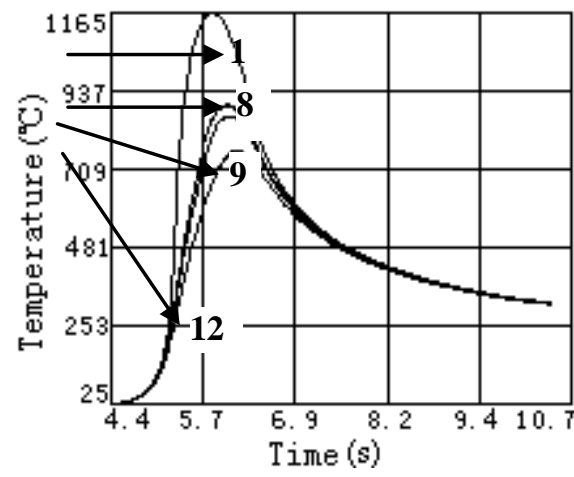

Fig.6 Varying relationship between temperature and time of element 1, 8, 9 and 12

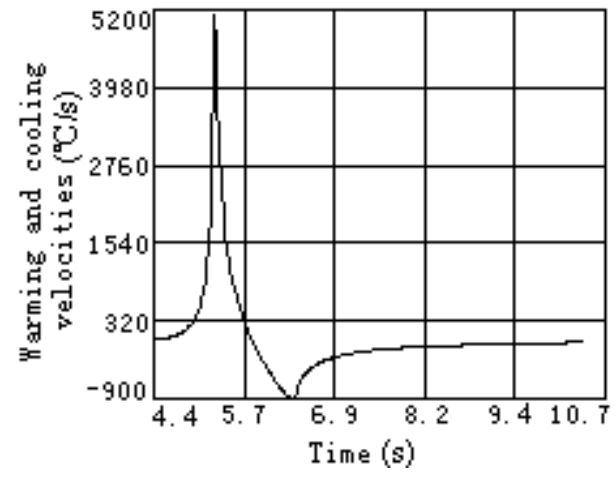

Fig.7 Varying relationship between warming and cooling velocities and time of element 1

Fig.8 shows the distribution of overcooling austenite with no decompose at 7.3s, and Figure.9 presents the distribution of martensite at room temperature (there is still some residual austenite that has the same distribution shape as Figure.8, with the maximal content of 3.5\%). It can be seen from the results that there is no $\mathrm{F}+\mathrm{P}$ and $\mathrm{F}+\mathrm{P}+\mathrm{B}$, indicating that the cooling velocities of overcooling austenite in the two regions are so quick that the incubation periods haven't finished; in the bainite region, the incubation period has finished and a small amount generates; the distribution shape of bainite is similar to Fig. 8 and the maximal content is only $0.6 \%$, so it can be ignored. 


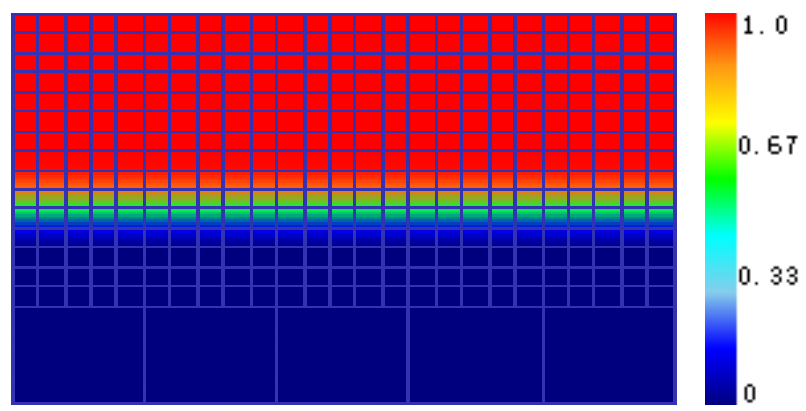

Fig.8 Distribution of overcooling austenite at 7.3s

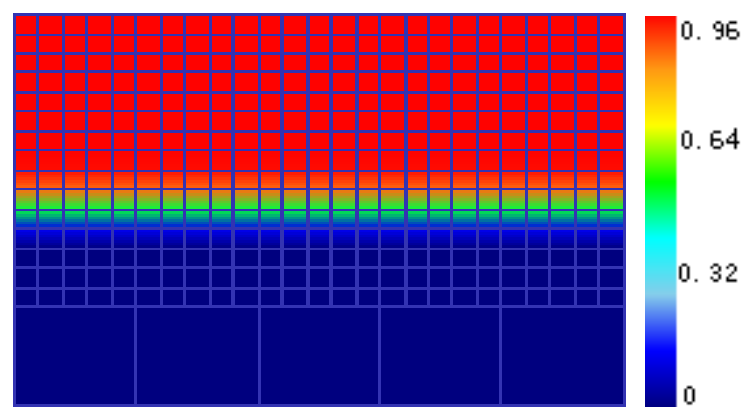

Fig.9 Distribution of martensite at room temperature

When having cooled down to room temperature, in the direction of depth, the grinded surface layer can be divided into three regions according to microstructure: the region between 0 and $0.8 \mathrm{~mm}$ contains mainly martensite that has a stable content of $96 \%$, besides, there existing little residual austenite, called the entirely hardened zone; the content of phases in the region between $0.8 \mathrm{~mm}$ and $1.2 \mathrm{~mm}$ varies fiercely, where martensite decreases sharply from $94 \%$ to 0 and tempered sorbite increases steeply from $2 \%$ to $100 \%$, besides, there existing little residual austenite, called the transition zone; the region below $1.2 \mathrm{~mm}$ is called the basal body zone, because there is no phase transformations happened. The simulation results of microstructure coincide well with that observed by the SEM [6].

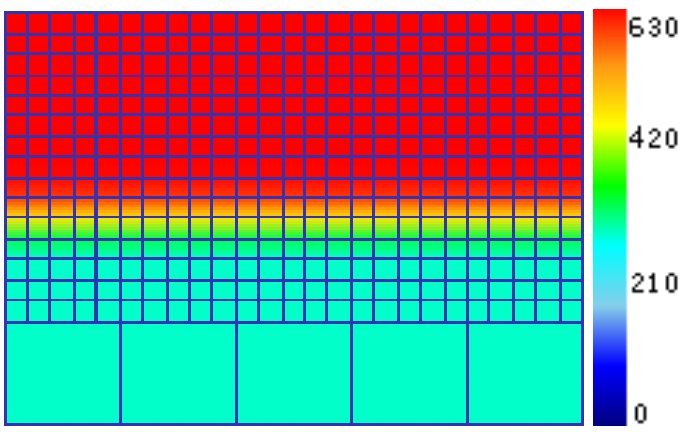

Fig.10.Micro-hardness field at room temp erature $\left(\mathrm{HV}_{0.5}\right)$

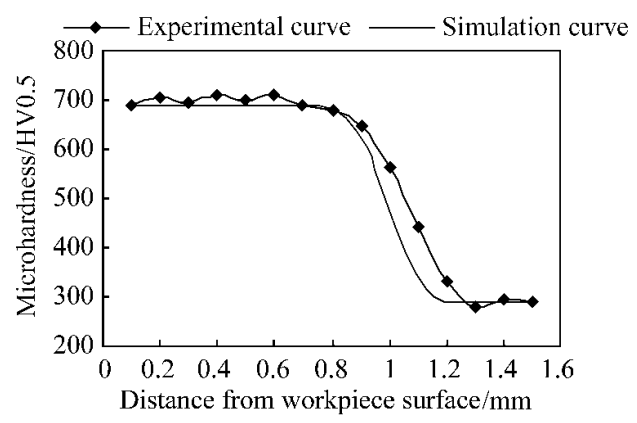

Fig.11. Experimental and simulation of micro-hardness distribution in the hardened layer

\section{Distribution and Alteration of the Micro-hardness Field}

Fig.10 is the micro-hardness field at room temperature and Fig.11 the experimental and simulated micro-hardness of the grinded surface layer. It can be seen that the surface layer of workpiece obtained excellent hardness stability: the region between 0 and $0.8 \mathrm{~mm}$ is the high hardness zone with stable hardness of $630 \mathrm{HV}_{0.5}$; the one between $0.8 \mathrm{~mm}$ and $1.2 \mathrm{~mm}$ is the hardness drop zone; the one below $1.2 \mathrm{~mm}$ is the low hardness zone with matrix hardness of $289 \mathrm{HV}_{0.5}$. The simulation curve coincides well with the experimental one, indicating that the simulation results are credible. 


\section{Summary}

With the moving of heat source, elements in the surface layer of workpiece experience six stages, namely slowly warming $\rightarrow$ quickly warming $\rightarrow$ slowly warming $\rightarrow$ slowly cooling $\rightarrow$ quickly cooling $\rightarrow$ slowly cooling. To elements in the loading layer, the average warming velocity, instantaneous maximal warming velocity and instantaneous maximal cooling velocity are $814^{\circ} \mathrm{C} / \mathrm{s}, 5150^{\circ} \mathrm{C} / \mathrm{s}$ and $-900^{\circ} \mathrm{C} / \mathrm{s}$ respectively.

When having cooled down to room temperature, in the direction of depth, the grinded surface layer can be divided into three regions according to microstructure, namely the entirely hardened zone, the transition zone and the basal body zone.

The grinded surface layer can obtain excellent hardness stability: the region between 0 and $0.8 \mathrm{~mm}$ is the high hardness zone with stable hardness of $630 \mathrm{HV}_{0.5}$; the one between $0.8 \mathrm{~mm}$ and $1.2 \mathrm{~mm}$ is the hardness drop zone; the one below $1.2 \mathrm{~mm}$ is the low hardness zone with matrix hardness of $289 \mathrm{HV}_{0.5}$.

\section{Acknowledgement}

This research is based upon work by the NNSF of China (Grant No.51075192), National key projects of P. R. China (Grant 2013ZX04009031) and Nantong key laboratory (CPI2014002).

\section{References}

[1] Ju-Dong LIU , Gui-Cheng WANG, Kang-Min CHEN, et al, China Mechanical Engineering. 16(2005) 1013-1017. (In Chinese)

[2] Brinksmeier E, Brockh off T. Annals of the CIRP. 45(1996) 283-286.

[3] Brockhoff T, Grinding-Hardening. Annals of the CIRP, 48(1999):255-260.

[4] I. Zarudi, L.C. Zhang. J. MATER. Sci. 37(2002) 3935-3943.

[5] I. Zarudi, L.C. Zhang. J. MATER. Sci. 37(2002) 4333-4341.

[6] Ju-Dong LIU, Studies on the Forming Mechanism of the Grinding-Hardening and Its Application Fundament, Ph.D. Dissertation, Jiangsu University, Zhenjiang, China, 2005.(In Chinese)

[7] Song-Wei HUANG, Ju-Dong LIU. Tool Engineering. 45(2011)75-77. (In Chinese)

[8] Ke-Ming LIU, Zhuang MA, Lian-Yong ZHANG.MATER MECH ENG. 36(2012) 58-61.(In Chinese)

[9] Zhuang MA, Bo LIU, Hai-Fang SHI.ORDNANCE MATER SCI ENG. 34(2011)26-28.

[10] W.B. Rowe, M.N. Morgan, S.C.E. Black. Annals of the CIRP. 47(1998) 275-279. 